\title{
Fuzzy-Clustering Based Cost Modeling of Disassembly Planning for EOL Products
}

\author{
Zhou Ziqiang $^{*}{ }^{* 1,2}$, Dai Guohong ${ }^{1,2}$, Wu Zhaoren ${ }^{2,3}$ and Zhang Xiangyan ${ }^{2,3}$ \\ ${ }^{I}$ School of Mechanical Engineering, Changshu Institute of Technology, Changshu, Jiangsu, 215500, P.R. China \\ ${ }^{2}$ Jiangsu Key Laboratory of Recycling and Reuse Technology for Mechanical and Electronic Products (Changshu \\ Institute of Technology), China \\ ${ }^{3}$ School of Mechatronic Engineering, China University of Mining and Technology, Xuzhou 221116, P.R. China
}

\begin{abstract}
Cost model is a key issue in the disassembly process planning, because the optimized disassembly sequence is determined by ranking several possible disassembly operations. In this study, a fuzzy cost model for disassembly processes was developed based on the fuzzy clustering method. The objective was to solve the problems in the practical applications of currently used quantitative models of disassembly cost model, which is based on the change times of the tools operation or the disassembly time. The sample data were obtained through the disassembly tests of the typical EOL (end-of-life) products. Following this, the transitive closure operations were performed after standardization and normalization. Dynamic clustering was carried out on the basis of above results, and appropriate clustering results were selected to construct the membership function of fuzzy costs. This study also proposed a method of proportional interpolation to expand the directly built membership function to the uncovered discourse domain, resulting in more practical fuzzy cost models. Finally, the disassembly process of a general reducer was adopted as an example to verify the feasibility of the above method.
\end{abstract}

Keywords: Disassembly, Disassembly cost model, Fuzzy clustering, Recycling, Remanufacturing.

\section{INTRODUCTION}

The disassembly and recycling of EOL products have attracted increased attention. Reasonable disassembly and recycling of EOL products can not only reduce environmental pollution, but also significantly reduce the consumption of natural resources. Therefore, there have been lots of studies on the disassembly theory of EOL products in recent years. H.Srinivasan and R.Gadh presented a method of wave propagation for modeling the single selective disassembly [1]. Following this, Jianjun Yi, et al. improved this method. A geometric algorithm was taken into account for disassembly strategy [2]. Lu Zhong, et al. put forward a method of Component-fastener graph to model the structure of product [3]. Shana Smith, et al. presented a method of disassembly sequence structure graph based on the matrix of contact constraint and motion constraint [4]. Ying Tang et al. presented a fuzzy petri net based approach to construct the model EOL product and obtain the optimal disassembly sequence [5]. Ahmed Elsayed et al. used the bill of material $(\mathrm{BOM})$ and genetic algorithm to generate the near optimal disassembly sequence for end-of-life electronic products [6]. The current strategic research on disassembly mainly focused on finding an optimal sequence for disassembly, so that the valuable parts of EOL products can

*Address correspondence to this author at the School of Mechanical Engineering, Changshu Institute of Technology, Changshu, Jiangsu, 215500 Chang; Tel: 86-512-52251595; Fax: 86-512-52251595;

E-mail: zzq_hefei@163.com be reused or remanufactured and the rest is recycled as material.

In order to compare different disassembly sequences, disassembly cost is a matter of main concern. Usually, the least-cost path is selected as the optimal path among various disassembly sequences. In current studies, the disassembly time or the change times of the tool operation pose is mostly adopted as the quantitative model of disassembly cost. S.Kara et al. used disassembly time of each operation as cost model to determine the optimal disassembly sequence [7]. Shana $\mathrm{S}$ et al. used the number of direction change, number of components and fasteners which needed to be resolved as cost model for disassembly sequence planning [8]. Li Hsing et al. proposed a cost model which included disassembly time, cost of disassembly equipment and labor to determine the optimal disassembly sequence [9]. T.F. Go et al. also useddisassembly time as cost model, furthermore, they proposed a penalty index for direction change [10].

The above mentioned cost model depends on the disassembly time or tools direction change method which is feasible for simple products, but would be more difficult to be used for EOL products with complex structure and large number of parts, because not only simple hand tools, but also various electric and hydraulic tools need to be used in the disassembly of EOL products. The change times of the tool make it difficult to represent the disassembly cost. In addition, the disassembly time for the same component may be different due to uncertainty in the joint strength between parts resulting from corrosion, clogging or other reasons. 
Therefore, accurate quantification models are inappropriate for the modeling of disassembly cost. In this study, the difficulty degree of the disassembly cost representation was denoted using fuzzy variable based on the fuzzy theory, which can be easily accepted by disassembly operators. Therefore, it was needed to build fuzzy cost models to adapt to the features of EOL products, which use fuzzy sets to denote the difficulty in disassembly with different joints.

According to fuzzy theory, the common methods used to build fuzzy sets include, expert experience method, statistical test method, and minimum fuzzy method. However, there are some disadvantages involved in using these methods for building the fuzzy sets of disassembly cost. For example, major errors may occur due to the subjective intuition of the expert experience method, while large amounts of data samples are required for the statistical test method and minimum fuzzy method. In this study, a modeling method of disassembly cost based on fuzzy clustering was proposed. Firstly, a small number of samples were collected based on the disassembly of typical EOL products, which were analyzed using fuzzy clustering method. Furthermore, fuzzy sets of the disassembly cost were constructed according to the clustering results.

\section{FUZZY COST MODEL FOR DISASSEMBLY PROCESS PLANNING}

The disassembly sequence planning based on fuzzy disassembly cost is shown in Fig. (1). Firstly, a small number of samples were extracted from a type of EOL products to carry out disassembly tests. During the disassembly process, the disassembly torque and energy consumption, etc., were measured using torque wrench, current power meter and other tools, or otherwise using sensors installed in tools. After disassembly, the obtained data were used for fuzzy clustering analysis. Finally, according to the results of dynamic clustering, appropriate cluster state was selected to build fuzzy sets of disassembly cost.

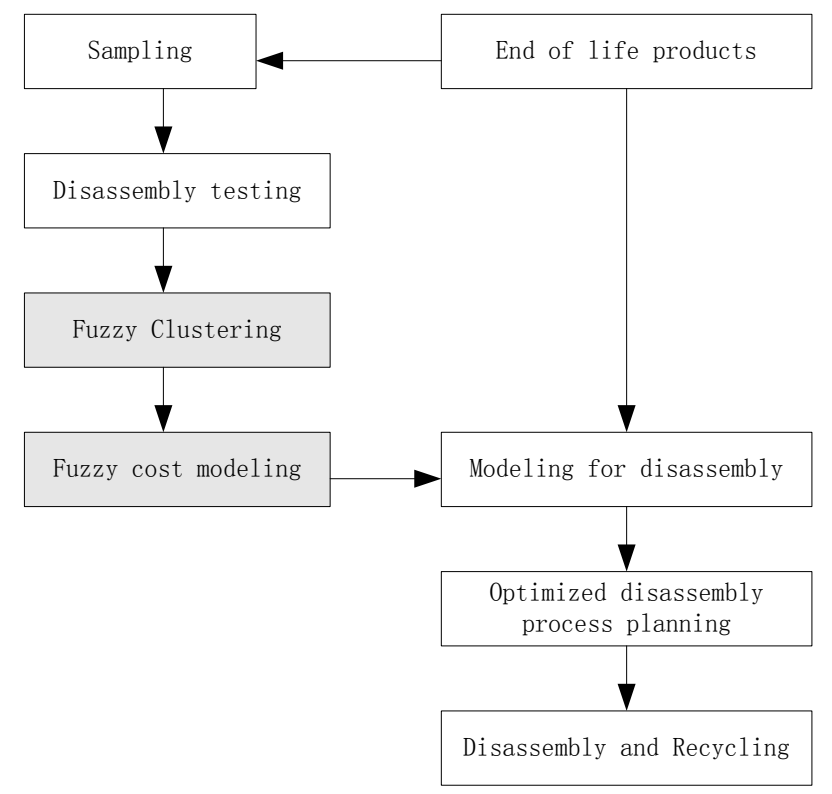

Fig. (1). Procedure of fuzzy cost based disassembly process planning.
After the completion of the fuzzy sets of disassembly cost, an appropriate searching program for the optimal disassembly sequence can be adopted to solve the disassembly and recycling strategies of EOL products. There are already many such algorithms and procedures for this purpose, which can be used for the disassembly process planning. Ahmed ElSayed et al. applied the genetic algorithm in disassembly sequence for EOL products [11]. J.F. Wang et al. used ant colony algorithm to search optimal disassembly sequence in disassembly process planning [12]. Elif Kongar et al. presented a genetic algorithm to determine the best disassembly sequence [13]. By L.M. et al. implemented a genetic algorithm based fuzzy logic approach for disassembly process [14]. Wei-Chang Yeh et al. presented a revised simplified swarm optimization algorithm for disassembly sequencing problem [15]. Belarmino et al. presented an efficient GRASP algorithm for disassembly sequence planning [16].

\section{PROCEDURE OF FUZZY CLUSTERING WITH SAMPLING DATA}

The fuzzy clustering process of sample data obtained from disassembly tests are shown in Fig. (2). Firstly, a sample data list needs to be established. Generally, each row of the table represents a group of sample data, i.e., all the data collected in a disassembly process for particular joint. Each column is a corresponding test indicator, such as the dismounting force, the energy consumption, and time. Following this, the sample data list is standardized. The purpose of standardization is to eliminate the influence of different dimensions due to different indicators. The maximum and minimum method is used here (as shown in Eq. 1), where, maxj and minj are the maximum and minimum values of column $\mathrm{j}$ (the $\mathrm{jth}$ indicator), respectively. Advantage of this method not only involves excluding the effect of dimensions, but also avoiding the submergence of the role played by the minimum value. By this means, all the data are compressed into the region of $[0,1]$.

$$
X_{i j}=\frac{\mathrm{A}_{i j}-\min _{j}}{\max _{j}-\min _{j}},(j=1,2,3,4)
$$

In order to build a similarity relationship between disassembly operations, a similarity relationship matrix is established. The maximum and minimum approaching method (shown in Eq. 2) is used for calculation, in which $X_{i, k}$ is the element value of row $i$ and column $j$ after the standardization of original materials; materials; $\vee$ and $\vee$ are the maximum and minimum Zadeh operators respectively.

$$
R_{i j}=\frac{\sum_{i=1}^{n}\left(X_{i k} \wedge X_{j k}\right)}{\sum_{i=1}^{n}\left(X_{i k} \vee X_{j k}\right)}, k=(1,2,3 \cdots 11)
$$

After the above calculation, the similarity relationship matrix $R_{i j}$ can be obtained. $R_{i j}$ is used to indicate the association degree between various constraints, i.e., determining the interrelated association between disassembly operations. On this basis, fuzzy clustering can be carried out. Therefore, the similarity matrix is the constraint correlation 
function. The element ri,j in the similarity matrix represents the similarity degree between the disassembly operations Di and $\mathrm{Dj}$; and ri, $=0$ denotes that they are completely different; while $\mathrm{ri}, \mathrm{j}=1$ denotes that they are the same and the diagonal elements of this matrix are all equal to one.

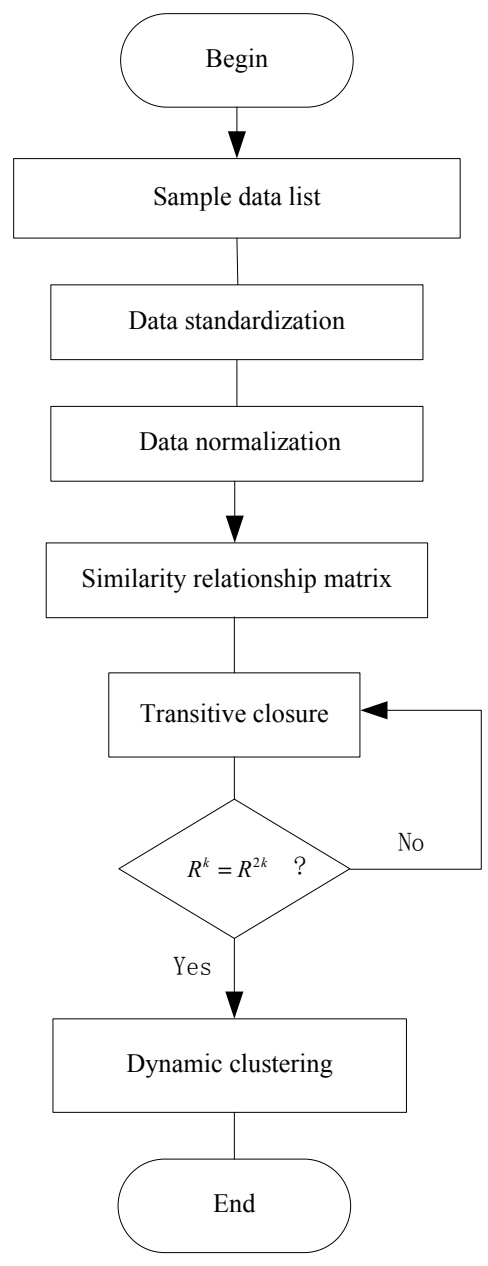

Fig. (2). Process of fuzzy clustering for sample data.

The transitivity must be satisfied for the clustering of the matrix $R_{i j}$, i.e., in which $R \circ R$ is contained in $\mathrm{R}$. The symbol。 denotes the convolution operation. In order to quickly solve the transitive closure $t(R)$, the square algorithm, which is mostly commonly used, has been adopted in this study. Namely, by calculating the relationship sequence as follows until no new relationship was generated, $R^{k} \circ R^{k}=R^{k}$ was obtained. The transitive package was $t(R)=R^{k}$.

$R \rightarrow R^{2} \rightarrow R^{4} \rightarrow \cdots \rightarrow R^{2 k}=t(R)$

Finally, different confidence levels i.e., the threshold $\lambda$ can be selected. The above transitive closure was clustered, resulting in a corresponding logical matrix $R^{\prime}$. The approach is shown in Eq. (4), where, $r_{i j}^{\prime}$ is the element in $R^{\prime}$.

$r_{i, j}^{\prime}=\left\{\begin{array}{ll}0 & r_{i, j} \leq \lambda \\ 1 & r_{i, j} \geq \lambda\end{array} \quad(i, j=1,2 \cdots, n)\right.$
There are only two cases including 0 and 1 for the elements in $R^{\prime}$. By selecting different thresholds $\lambda$, a series of logical matrixes $R^{\prime}$ can be obtained. Using appropriate program tools such as Matlab, the corresponding dynamic clustering map can be drawn. Thereby, the change of $\lambda$ and the clustering state of the sample data can be easily observed. After that, an appropriate cluster state can be selected according to the size of fuzzy sets, which can be used to establish fuzzy sets of disassembly cost.

\section{FUZZY COST MODELING FOR DISASSEMBLY PROCESS PLANNING}

Fuzzy cost models in the disassembly process are represented by fuzzy sets. Namely, the linguistic variables such as "very difficult", "difficult", "medium", "easy" and "very easy" are used to describe the ease degree of disassembly. Following this, the total cost of various disassembly sequences was determined by computing the fuzzy variables using the disassembly sequence planning algorithm, for addition, multiplication, size comparison, etc., resulting in an optimal disassembly sequence.

In order to create a fuzzy set according to the above clustering results, the form of the membership function must be determined first. Although there are several types of membership functions, the triangular membership function has been widely applied in engineering due to simple form and convenience in use. The triangular membership function is also used in the fuzzy modeling of disassembly cost. The form of triangular membership functions is shown in Fig. (3). It is represented by a ternary array $(\mathrm{L}, \mathrm{M}, \mathrm{N})$, where, $\mathrm{L}$ is the minimum value of the domain which represents the linguistic variables; $M$ represents the domain value with the largest membership value; and $\mathrm{N}$ is the maximum value of the domain, which represents the linguistic variables.

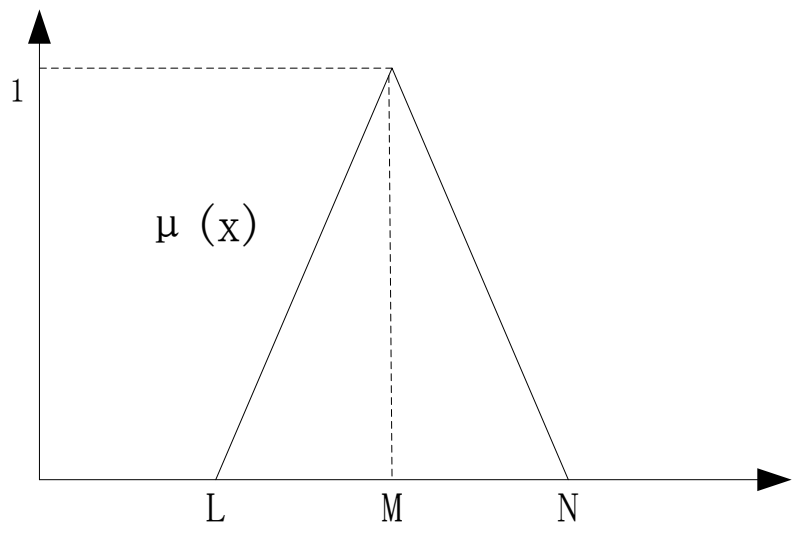

Fig. (3). Triangular membership function.

According to the clustering results obtained in the previous section, the centroid method can be used to calculate the center of each data cluster, namely the domain value where the point $M$ is located.

$v_{k}^{j}=\frac{1}{n_{j}} \sum_{j=1}^{n_{j}} x_{i, k}^{j}(k=1,2,3,4)$ 


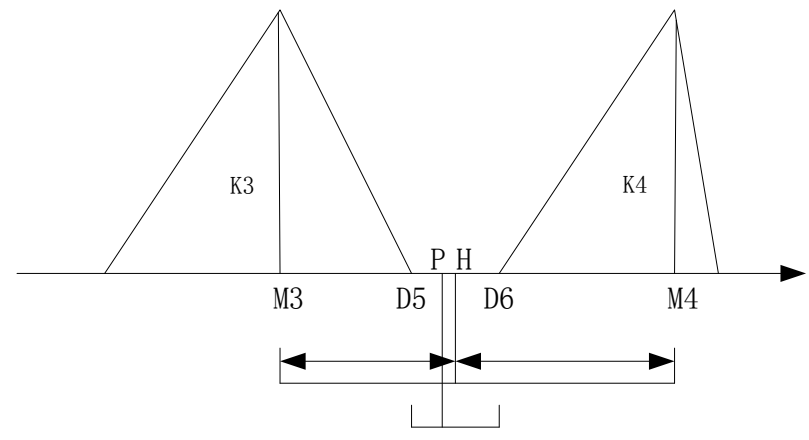

Fig. (4). Proportional interpolation.

Following this,, the minimum and the maximum values in each set of data were used to replace the above $\mathrm{L}$ and $\mathrm{M}$, and the domain distribution of the corresponding linguistic variables could be obtained. However, the developed fuzzy cost model should be able to cover all possible domain regions for the EOL products otherwise, the data may be lost due to the discontinuity of the fuzzy domains in the fuzzy processing of particular connection relationships. As shown in Fig. (4), two fuzzy linguistic variables K3 and K4 constructed using the method described above were not included in the regions where the domains D5 and D6 were located. If the domain values in this region are treated as input, appropriate language variable cannot be generated. Therefore, this paper proposed a method of proportion interpolation. Firstly, the midpoint of the uncovered domain region was calculated, i.e., the domain value of the midpoint $\mathrm{H}$ between D5 and D6. Following this, the interpolation point $\mathrm{P}$ was calculated according to the following proportional relationship.

$\frac{M_{3} H}{H M_{4}}=\frac{D_{5} P}{P D_{6}}$

After the reprocessing of the domain values for linguistic variables, a fuzzy cost model of disassembly process was obtained to meet the actual requirements.

\section{CASE STUDY}

In this study, the disassembly process of a common reducer was adopted as an example for fuzzy cost modeling. The reducer has different structural forms, including onelevel reducer, two-level reducer, single output and dual output. But the joint modes of its components are roughly the same, and therefore they generally belong to the same category of products. For modeling convenience, a small and medium-sized one-level reducer was selected as the typical product for disassembly testing. The structure of this onelevel reducer is shown in Fig. (5).

Disassembly was operated by special disassembly tools and testing tools for main components. Four indicators including disassembly duration, disassembly moment, disassembly energy consumption, and tool cost were selected. Among them, the tool cost is the cost of each operation calculated from the tool purchase price and service life. Eleven data sets were obtained by testing, and the data sample list is shown in Table $\mathbf{1}$.

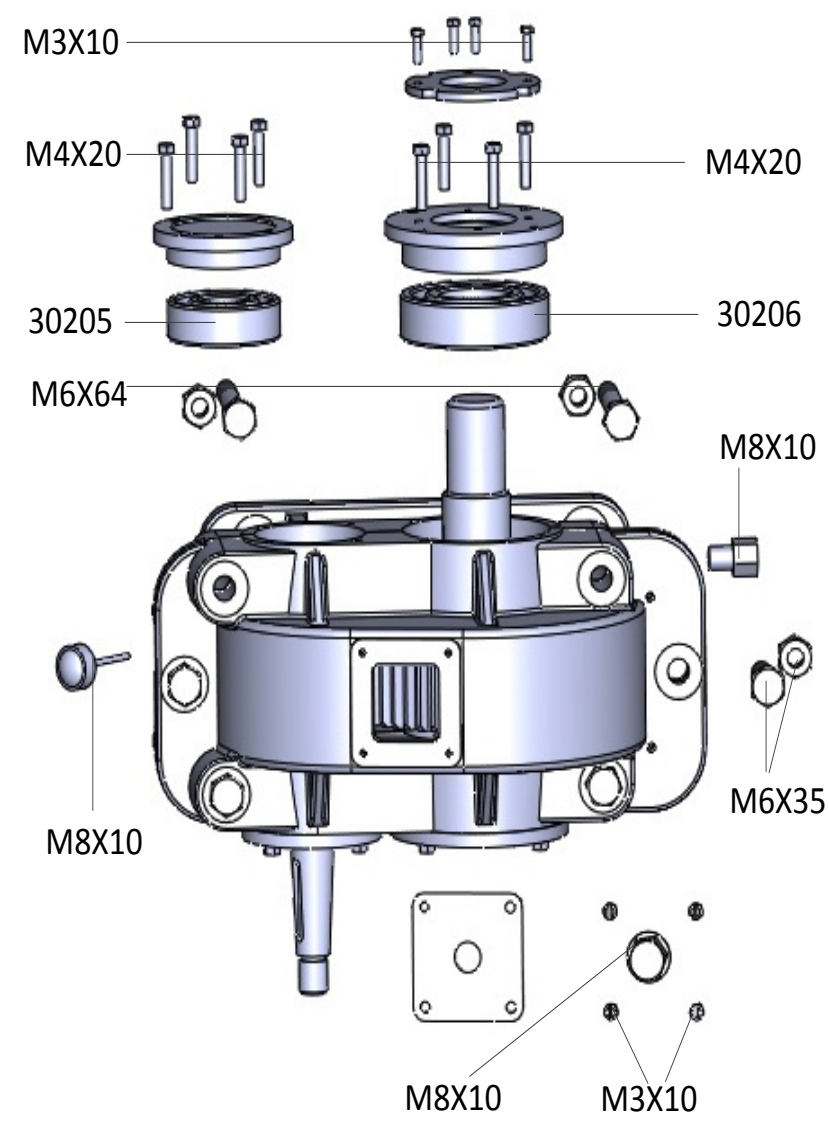

Fig. (5). The disassembly structure of reducers.

The data list was standardized and normalized according to the above method, and the following similarity relationship matrix was obtained. Following this, the transitive closure was calculated for the similarity relationship matrix $\mathrm{R}$, and the obtained transitive closure was :

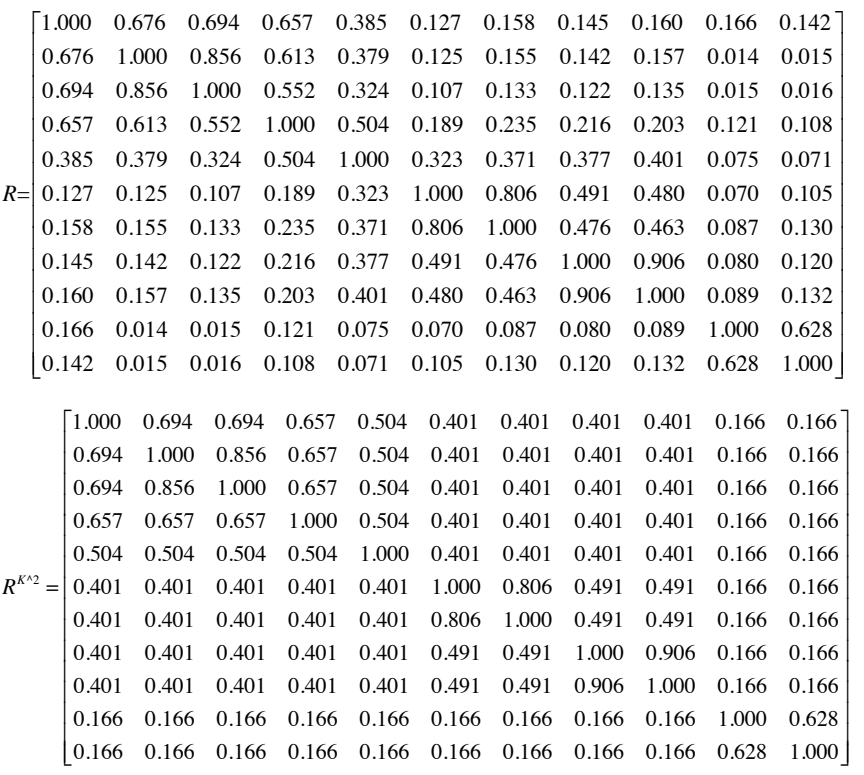

The diagonal elements of the transitive closure matrix were all one, and the matrix was symmetric. Therefore, only the upper half or the lower half of them needed to be investigated. The element values in the bottom line were 
Table 1. The sample data list of disassembly.

\begin{tabular}{|c|c|c|c|c|c|c|}
\hline \multicolumn{2}{|c|}{ Disassembly Operation } & $\boldsymbol{I}_{\mathbf{1}}$ & $\boldsymbol{I}_{\mathbf{2}}$ & $\boldsymbol{I}_{\mathbf{3}}$ & $\boldsymbol{I}_{\mathbf{4}}$ & Component Name \\
\hline \hline No. & Disassembly Part & Disassembly Time/ SD & Disassembly Torque/ N·M & Energy Consumption/ J & $\begin{array}{c}\text { Tool } \\
\text { Costs/ } \mathbf{T}\end{array}$ \\
\hline$D_{1}$ & $\mathrm{M} 8 \times 10$ & 5.8 & 1.25 & 125.6 & 8 & Oil level indicator \\
\hline$D_{2}$ & $\mathrm{M} 3 \times 10$ & 7.2 & 1.25 & 157 & 5 & Felt blade screw \\
\hline$D_{3}$ & $\mathrm{M} 3 \times 10$ & 6.6 & 1 & 125.6 & 5 & Observation window positioning screw \\
\hline$D_{4}$ & $\mathrm{M} 8 \times 10$ & 10 & 1.50 & 113.04 & 8 & Observation window air hole bolt \\
\hline$D_{5}$ & $\mathrm{M} 8 \times 10$ & 8.9 & 7 & 1780.38 & 42 & Fastening Bolt 1 of upper and lower tank \\
\hline$D_{6}$ & $\mathrm{M} 6 \times 64$ & 23.7 & 6.75 & 1318.8 & 33 & Fastening Bolt 2 of upper and lower tank \\
\hline$D_{7}$ & $\mathrm{M} 6 \times 35$ & 20.7 & 14 & 2512 & 18 & End cover (through cover) positioning screw \\
\hline$D_{8}$ & $\mathrm{M} 4 \times 20$ & 10.9 & 13 & 2332.57 & 18 & End cover (stuffy cover) positioning screw \\
\hline$D_{9}$ & $\mathrm{M} 4 \times 20$ & 8.3 & 24.5 & 13 & Clearance Fit 1 of tapered roller bearings \\
\hline$D_{10}$ & 30205 & 1.2 & 37.37 & 17 & Clearance Fit 2 of tapered roller bearings \\
\hline$D_{11}$ & 30206 & 1 & 0.34 & & 8 & \\
\hline
\end{tabular}

adopted as the basis value for the confidence level. The corresponding logical matrixes were calculated in ascending order, and the dynamic clustering chart was created using Matlab, as shown in Fig. (6).

According to the general requirements of the disassembly process, it is reasonable to classify the difficulty for the component disassembly into five levels, which maintains a certain degree of differentiation. The language variables "very low" (VL), "low" (L), "moderate" (M), "high" (H), "very high" (VH) were used to represent the disassembly costs. Namely, when the confidence level $\lambda$ located in the region was $[0.504,0.657]$, the sample data were clustered into five categories: $\{\mathrm{D} 5, \mathrm{D} 9\},\{\mathrm{D} 6, \mathrm{D} 7\},\{\mathrm{D} 5\},\{\mathrm{D} 1, \mathrm{D} 2$, D3, D4 $\}$ and $\{$ D10, D11 $\}$.

Finally, the triangular membership functions were built for the above aggregate of data using centroid method and proportion interpolation which was extended to the uncovered domain regions. The final fuzzy cost set model was obtained, and its membership function is shown in Fig. (7). Fig. (8) shows the fuzzy cost set model constructed by the expert experience method [17]. It can be seen that there were obvious differences between the two results and the fuzzy set obtained by fuzzy clustering is more practical.

\section{CONCLUSION}

At present, the cost model based on the change times of the tool operation pose and the disassembly time was difficult to apply. The disassembly cost described by fuzzy linguistic variables is easier to estimate in engineering applications. However, it is critical to establish a disassembly cost model based on fuzzy domain according to the specific features of the EOL products. The expert experience method in fuzzy theory often leads to big deviation in fuzzy set due to the subjective errors and incomplete experience. The statistical method requires a lot of sample data. In this study, the disassembly tests were conducted for some EOL products. Fuzzy clustering analysis was carried out using the obtained test data, and the

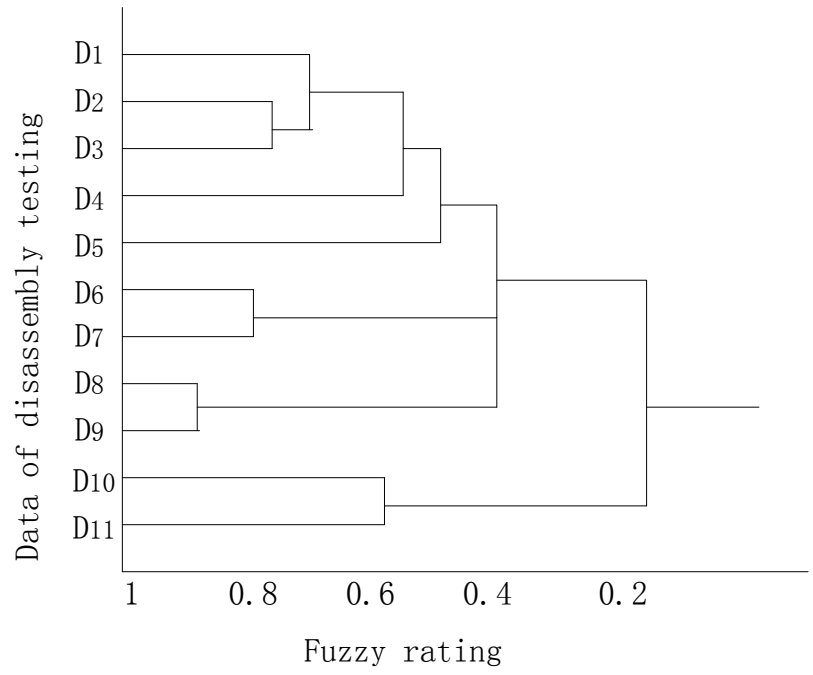

Fig. (6). Dynamic clustering chart of fuzzy clustering.

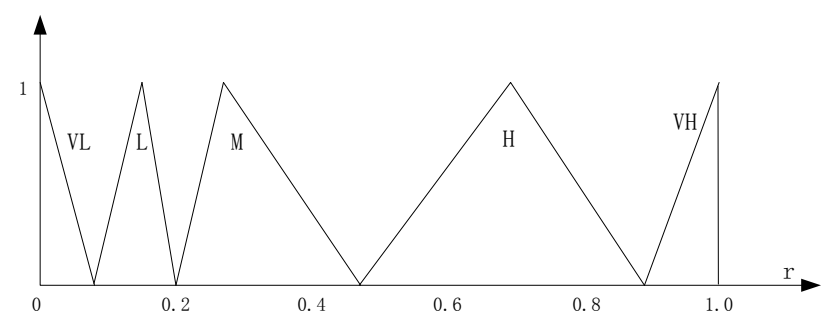

Fig. (7). Fuzzy cost model by fuzzy clustering.

membership function of the disassembly cost was built up. The proportion interpolation method was proposed to expand the domain regions uncovered by the sample data. This method is simpler and more practical. Considering the engineering applications, if specific software tools can be developed to allow the disassembly operator to directly input the sample data and generate a data model of disassembly cost, it would make this method more feasible. 


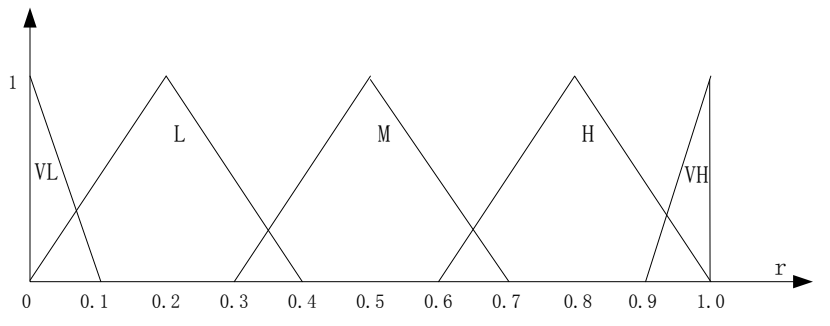

Fig. (8). Fuzzy cost model by fuzzy clustering.

\section{CONFLICT OF INTEREST}

The authors confirm that this article content has no conflict of interest.

\section{ACKNOWLEDGEMENTS}

This work was financially supported by the 333 High Level Talent Engineering of Jiangsu Province of China (No.BRA2012158) and Jiangsu Science \& Technology Pillar Program of China (No. BE2013060).

\section{REFERENCES}

[1] H. Srinivasan, and R. Gadh, "A geometric algorithm for single selective disassembly using the wave propagation abstraction", Computer Aided design, vol. 30, no. 8, pp. 603-613, 1998.

[2] J. Yi, B. Yu, and L. Du, "Research on the selectable disassembly strategy of mechanical parts based on the generalized CAD model", International Journal of Advanced Manufacturing Technology, vol. 37, no. 5, pp. 599-604, 2008.

[3] L. Zhong, S. Youchao, and W. Haiqiao, "Disassembly sequence planning for maintenance based on metaheuristic method", Aircraft engineering and aerospace technology: an International Journal, vol. 83, no. 3, pp. 138-145, 2011.

[4] S. Smith, and G. Smith, "Disassembly sequence structure graphs: An optimal approach for multiple-target selective disassembly sequence planning, Advanced Engineering Informatics, Advanced Engineering Informatics, vol. 26, no. 4, pp. 306-316, 2012.

[5] Y. Tang, M. Zhou, and M. Gao, "Fuzzy-Petri-Net-Based Disassembly Planning Considering Human Factors", IEEE transactions on systems, man and cybernetics-part a: systems and human, vol. 36, no.7 pp. 718-726, 2006.

[6] A. El-Sayed, E. Kongar, and S. M. Gupta, "An Evolutionary algorithm for selective disassembly of end-of-life products, International Journal of Swarm Intelligence and Evolutionary computation, 2012, 1: 1-7.

[7] S. Kara, P. Pornprasitpol, and H. Kaebernick, "A selective disassembly methodology for end-of-life products", Assembly Automation, vol. 25 , no. 2 , pp. $124-134,2005$

[8] S. Shana, S. Smith, and W.-H. Chen, "Rule-based recursive selective disassembly sequence planning for green design", Advanced Engineering Informatics, vol. 25, no.5, pp. 77-87, 2011.

[9] L.-H. Shih and S.-C. Lee, "Optimizing disassembly and recycling process for EOL LCD-Type products: A heuristic method, IEEE Transactions On Electronics Packaging Manufacturing, vol. 30, no. 7, pp.213-220, 2007.

[10] T.F. Go, D.A. Wahab, M.N. Ab. Rahman, R. Ramli, A. Hussain, "Genetically optimised disassembly sequence for automotive component reuse", Expert Systems with Applications, vol.39, no. 11, pp. 5409-5417, 2012.

[11] A. El-Sayed, E. Kongar, and S. M. Gupta, "A genetic algorithm approach to end-of-life disassembly sequencing for robotic disassembly[C]. 2010 Northeast Decision Sciences Institute Proceedings.

[12] J.F. Wang, J.H.Liu, and S.Q.Li, "Intelligent selective disassembly using the ant colony algorithm", Artificial Intelligence for Engineering Design, Analysis and Manufacturing, vol. 17 pp. 325333, 2003.

[13] E. Kongar, and S. M. Gupta, "Disassembly sequencing using genetic algorithm", The International Journal of Advanced Manufacturing Technology, vol. 30, no.12, PP. 497-506, 2006.

[14] B. L.M. Galantucci, G. Percoco, and R. Spina, "Assembly and Disassembly Planning by using Fuzzy Logic \& Genetic Algorithms", International Journal of Advanced Robotic Systems, vol. 1, no. 1, pp. 67-74, 2004.

[15] W.-C. Yeh, C.-M. Lin, and S.-C. Wei, "Disassembly Sequencing Problems with Stochastic Processing Time using Simplified Swarm Optimization", International Journal of Innovation, Management and Technology, vol. 3, no. 6, pp. 226-231, 2012.

[16] B. Adenso-Diaz, S. Garciacarbajal, and S. Lozano, "An Efficient Grasp algorithm for disassembly sequence planning", $O R$ Spectrum, vol. 29, no. 1, pp. 535-549, 2006.

[17] Z. Zhou, G. Dai, and Z. Wu, "Research of Fuzzy cost model for selective disassembly planning", Advanced Materials Research, vol. 834 , no. 11, pp. 1732-1735, 2014 